ARTICLE

DOI: $10.1038 / s 41467-017-02039-5$

\title{
Thin single crystal perovskite solar cells to harvest below-bandgap light absorption
}

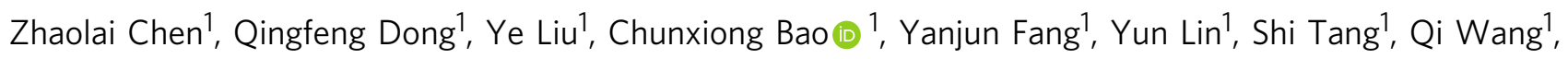
Xun Xiao ${ }^{1}$, Yang Bai ${ }^{1}$ Yehao Deng ${ }^{1} \&$ Jinsong Huang ${ }^{1,2}$

The efficiency of perovskite solar cells has surged in the past few years, while the bandgaps of current perovskite materials for record efficiencies are much larger than the optimal value, which makes the efficiency far lower than the Shockley-Queisser efficiency limit. Here we show that utilizing the below-bandgap absorption of perovskite single crystals can narrow down their effective optical bandgap without changing the composition. Thin methylammonium lead triiodide single crystals with tuned thickness of tens of micrometers are directly grown on hole-transport-layer covered substrates by a hydrophobic interface confined lateral crystal growth method. The spectral response of the methylammonium lead triiodide single crystal solar cells is extended to $820 \mathrm{~nm}, 20 \mathrm{~nm}$ broader than the corresponding polycrystalline thin-film solar cells. The open-circuit voltage and fill factor are not sacrificed, resulting in an efficiency of $17.8 \%$ for single crystal perovskite solar cells.

\footnotetext{
${ }^{1}$ Department of Mechanical and Materials Engineering, University of Nebraska, Lincoln, NE 68588, USA. ${ }^{2}$ Department of Applied Physical Sciences, University of North Carolina, Chapel Hill, NC 27599, USA. Zhaolai Chen and Qingfeng Dong contributed equally to this work. Correspondence and requests for materials should be addressed to J.H. (email: jhuang@unc.edu)
} 
O rganic-inorganic halide perovskites (OIHPs) have attracted tremendous attentions for solar cell application in the past few years ${ }^{1-9}$ due to the superior optoelectronic properties, such as large extinction coefficient ${ }^{10}$, long carrier recombination lifetime ${ }^{11}$, large carrier mobility ${ }^{12}$, unique defect physics ${ }^{13}$, etc. The efficiency of OIHP solar cells has reached $22.1 \%$ quickly after development in a few years by the improvement of material quality and device interface ${ }^{9}$, but it is facing a bottleneck caused by the non-optimized bandgap of the current perovskite materials. The most widely studied perovskite solar cells are based on $\mathrm{CH}_{3} \mathrm{NH}_{3} \mathrm{PbI}_{3}\left(\mathrm{MAPbI}_{3}\right)$, which has a bandgap too large for single-junction solar cells ${ }^{14}$. To reduce the bandgap of $\mathrm{MAPbI}_{3}$, composition engineering has been developed by partially replacing $\mathrm{MA}^{+}$and $\mathrm{Pb}^{2+}$ with $\mathrm{FA}^{+}$and $\mathrm{Sn}^{2+}$, respectively ${ }^{15-18}$, which however brings new challenges. The easy oxidation of $\mathrm{Sn}^{2+}$ to $\mathrm{Sn}^{4+}$ causes additional severe chemical instability of the Sn-containing perovskite ${ }^{17}$. Introduction of excessive $\mathrm{FA}^{+}$ions can cause the instability of material structure ${ }^{16}$.

Here we exploit an approach to broaden the absorption spectrum of perovskites by utilizing their below-bandgap transition. Below-bandgap absorption has been identified in $\mathrm{MAPbI}_{3}$ in previous studies, which was attributed to the indirect-bandgap absorption transition with a bandgap of $\sim 60 \mathrm{meV}$ smaller than the direct bandgap ${ }^{19-22}$. We found other perovskite materials, such as $\mathrm{MAPbBr}_{3}$, also showed below-gap absorption. Similar to silicon single crystal solar cells, further improvement of the device efficiency may be achieved by utilizing the below-bandgap absorption. However, the absorption coefficient corresponding to the below-bandgap transition is several orders magnitude smaller than that of the above-gap transition. Therefore, the thickness of the perovskite films thus needs to be over several micrometers $(\mu \mathrm{m})$ or more, which is however beyond the carrier diffusion length in existing polycrystalline perovskite films. Perovskite single crystals are shown to have much longer carrier diffusion length well above tens of micrometer due to the absence of grain boundaries and significantly reduced defect density ${ }^{23-26}$. Current studies of the intrinsic properties of the perovskites are mainly based on thick bulk single crystals with thickness of millimeter ${ }^{27,28}$ which are too thick for application in solar cells. Growing thin perovskite single crystals with large area represents an effective approach to overcome this obstacle ${ }^{29-31}$, however there is no effective method handling the micrometer-thick iodide-based perovskite single crystals with large area. Recently, Liu et $\mathrm{al}^{32}$ reported an efficiency of about $4 \%$ for perovskite single crystal solar cells. The low efficiency was due to the too large thickness of $50 \mu \mathrm{m}$ for the perovskite crystal and nonpassivated surface defects.

Herein, we report the growth of perovskite single crystals directly onto hole transport layer (HTL) covered indium tin oxide (ITO) substrates with controlled thickness of tens of micrometer and area of tens of millimeter square $\left(\mathrm{mm}^{2}\right)$. Solar cells based on the thin single crystals show obviously broader spectral response compared to the polycrystalline thin-film solar cells, while the open-circuit voltage $\left(V_{\mathrm{OC}}\right)$ and fill factor $(\mathrm{FF})$ of the solar cells remain comparable to those of polycrystalline thin-film solar cells, which demonstrates the potential for the application of perovskite single crystals to further boost the efficiency of perovskite solar cells.

\section{Results}

Thickness-dependent device performance. We first simulate the thickness-dependent absorption of $\mathrm{MAPbI}_{3}$ and device efficiency to find out the feasibility of increasing device efficiency by utilizing the below-bandgap transition in $\mathrm{MAPbI}_{3}$ and the details are shown in the "Method" section. Figure 1a shows an absorption coefficient curve of $\mathrm{MAPbI}_{3}$ from literature ${ }^{33}$, which does show two-four orders magnitude weaker absorption for below-bandgap transition than direct transition. Figure $1 \mathrm{~b}$ shows the calculated thickness-dependent percentage of light absorbed by the $\mathrm{MAPbI}_{3}$ films in devices where a full reflection of the light by a metal back electrode is assumed. When the thickness of $\mathrm{MAPbI}_{3}$ films increases from $500 \mathrm{~nm}$ to $200 \mu \mathrm{m}$, the absorption spectrum expands significantly to near-infrared region, leading to the redshift of the absorption edge to $850 \mathrm{~nm}$. The enhanced absorption in this wavelength range is important to increase the short-circuit current density $\left(J_{\mathrm{SC}}\right)$, because sunlight has a significant portion of
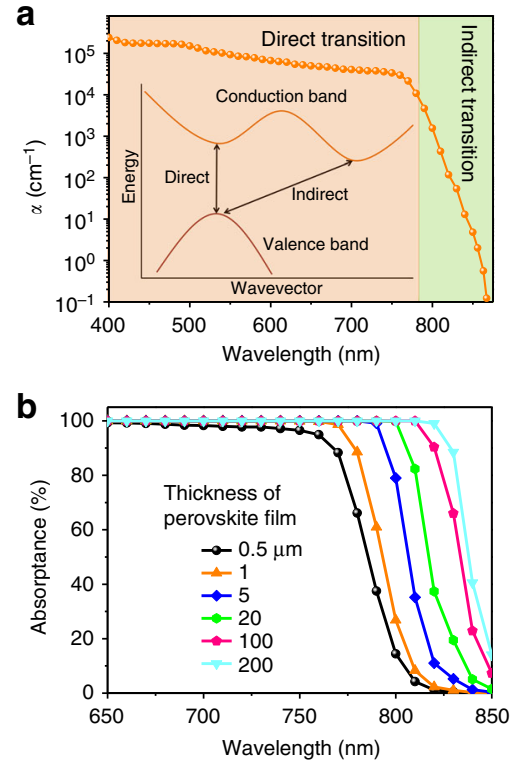

c

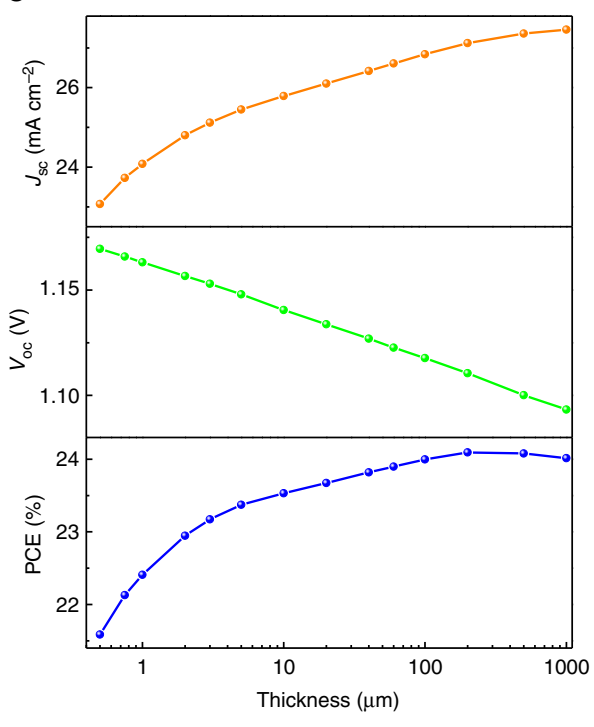

Fig. 1 Thickness-dependent perovskite absorption and device performance. a Schematic illustration of the direct, below-bandgap transitions and absorption coefficient of methylammonium lead triiodide $\left(\mathrm{MAPbl}_{3}\right)$ from a polycrystalline film. $\mathbf{b}$ The calculated absorption of $\mathrm{MAPbl}_{3}$ films with different thickness. c The calculated ideal dependence of $J_{\mathrm{sc}}, V_{\mathrm{oc}}$ and PCE of the single crystal solar cells on the thickness of the thin single crystals 


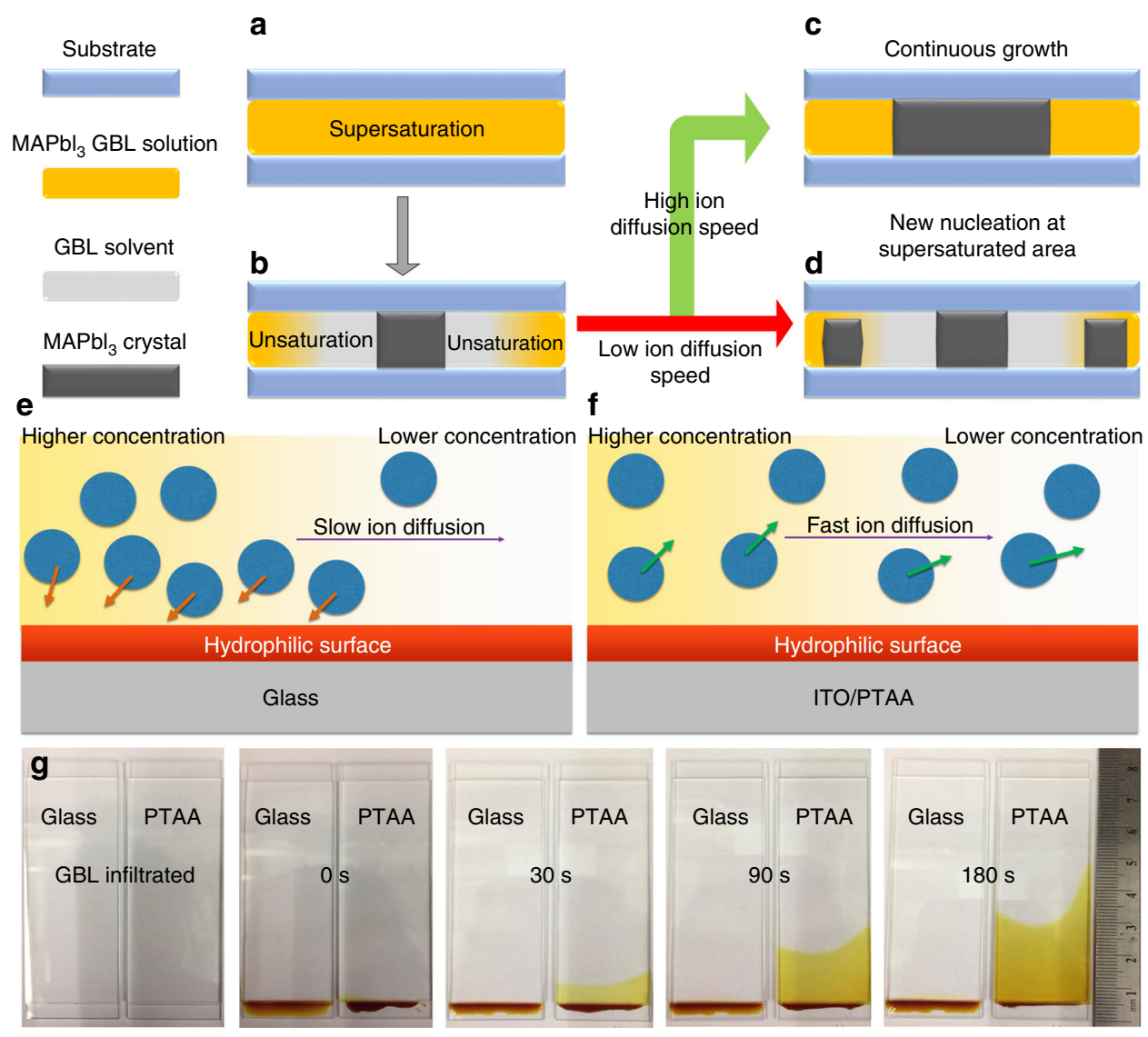

Fig. 2 Growth mechanism of perovskite thin single crystals. a-d Schematic illustrations of correlation between ion diffusion and thin single-crystal growth. Schematic illustrations of ion diffusion rate in the confined gaps using $\mathbf{e}$ hydrophilic and $\mathbf{f}$ hydrophobic substrates. $\mathbf{g}$ Photographs of the diffusion process of methylammonium lead triiodide $\left(\mathrm{MAPbl}_{3}\right)$ precursor solution in the confined gaps after different durations using hydrophilic glass and hydrophobic PTAA-covered ITO substrates

energy in this wavelength range. As shown in Fig. 1c, as the thickness of perovskite crystals increases from $500 \mathrm{~nm}$ to $200 \mu \mathrm{m}$, (JS) increases from $23.0 \mathrm{~mA} \mathrm{~cm}^{-2}$ to $27.1 \mathrm{~mA} \mathrm{~cm}^{-2}$ due to the broadened absorption spectrum. The $V_{\mathrm{OC}}$ of the devices, however, has an opposite variation trend with respect to the thickness of absorber based on the diode equation:

$$
V_{\mathrm{OC}}=\frac{k T}{q} \operatorname{In}\left(\frac{J_{\mathrm{SC}}}{J_{0}}\right)
$$

where $T, k, q$, and, $J_{0}$ are the absolute temperature, Boltzmann constant, elementary charge, and reverse bias saturated dark current density, respectively. The details for the simulation of crystal thickness-dependent efficiency limit of the single crystal solar cells can be found in the Supplementary Note. Though increased $J_{\mathrm{SC}}$ slightly increases $V_{\mathrm{OC}}$, larger thickness of perovskite films increases the charge recombination during their transport and thus $J_{0}$, which reduces $V_{\mathrm{OC}}$. The charge recombination dominates the variation of $V_{\mathrm{OC}}$ with a clear reduction with increasing crystal thickness, as shown in Fig. 1c. There should be an optimal absorber thickness that the perovskite single crystal solar cells reach the highest efficiency. To determine the optimal thickness of perovskite single crystals, we assumed a constant FF of $80 \%, 90 \%$ external quantum efficiency (EQE) over the whole absorption spectrum, which are all reasonable and are justified in the "Method" section. The power conversion efficiency (PCE) increases first and then decreases with increasing the thickness of single crystals, resulting in an optimal crystal thickness of $200 \mu \mathrm{m}$. When the crystal thickness is over $10 \mu \mathrm{m}$, the PCE is higher than
$23.5 \%$, which is far beyond the state-of-the-art values of $\mathrm{MAPbI}_{3}$ polycrystalline thin-film solar cells.

Growth and characterization of thin crystals. A diffusionfacilitated space-confined method was developed to obtain perovskite single crystals with controllable thickness down to micrometer scale. The confined space was constructed by two substrates (Fig. 2a), and the supersaturation of precursor solution was generated by the inverse solubility method ${ }^{23}$. The spaceconfined method was reported to grow thin perovskite singe crystals $^{31}$, however, the crystal thickness could hardly go down to $100 \mu \mathrm{m}$ for large-area perovskite crystals. The lateral crystal growth in this method can be limited by the inefficient long-range transportation of precursor ions along micrometer-size gap to continuously replenish the depleted precursors by crystal growth, which results in the formation of many small crystals or even polycrystals, as illustrated in Fig. 2d. When the gap is down to micrometer scale, the interaction between substrate surface and solvent in precursor solution becomes critically important for ion diffusion in solvent. Since the perovskite precursor ions generally form complex with the solvent ${ }^{34}$, the diffusion rate of precursor ions is determined by how fast the solvent molecules can transport on the substrate, and the surface tension determines how fast the solvent molecules close to the substrate can move along in-plane direction ${ }^{35-37}$. On wetting substrates, such as glass or ITO, the large surface tension imposes a friction force that drags down the ion diffusion ${ }^{38}$, as illustrated in Fig. 2e. Interestingly, when the substrates are covered with hydrophobic materials, such as PTAA $^{39}$, which is also a hole transport material 

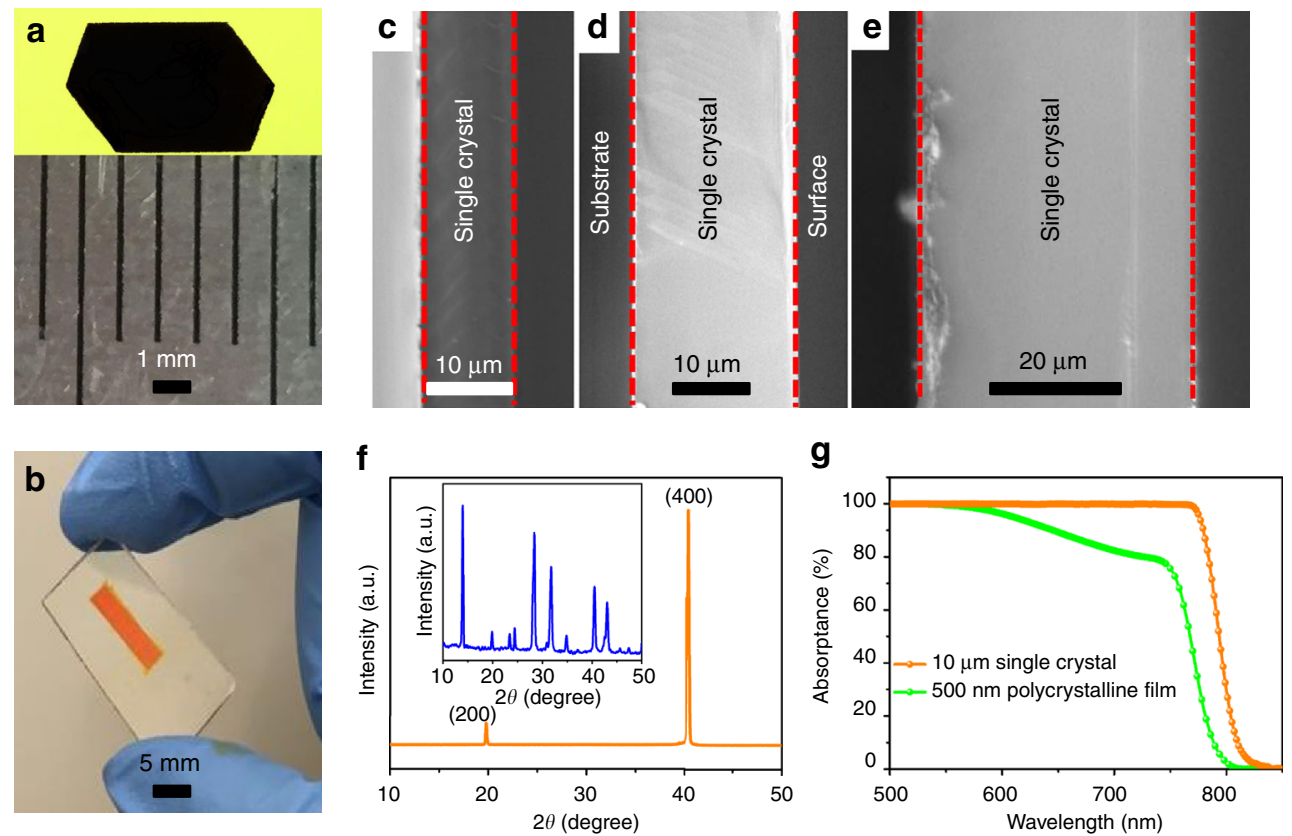

Fig. 3 Characterization of perovskite thin single crystals. Photographs of a methylammonium lead triiodide $\left(\mathrm{MAPbl}_{3}\right)$ thin single crystal and $\mathbf{b}$ methylammonium lead tribromide $\left(\mathrm{MAPbBr}_{3}\right)$ thin single crystal using the hydrophobic interface confined lateral growth method. Cross-sectional SEM images of the $\mathrm{MAPbl}_{3}$ thin single crystals with different thickness: $\mathbf{c} \approx 10 \mu \mathrm{m}, \mathbf{d} \approx 20 \mu \mathrm{m}, \mathbf{e} \approx 40 \mu \mathrm{m}$. $\mathbf{f}$ X-ray diffraction patterns of a MAPbl 3 thin single crystal and the grounded powder (inset). $\mathbf{g}$ Absorption spectra of a $10-\mu \mathrm{m}$-thick $\mathrm{MAPbl}_{3}$ thin single crystal and a 500 -nm-thick polycrystalline film

for perovskite solar cells, the ion diffusion is dramatically accelerated. The dramatically enhanced ion diffusion rate in micrometer-size gap enables a continuous growth of thin perovskite single crystals up to several millimeters along in-plane direction, as illustrated in Fig. 2c. To illustrate the dramatically enhanced ion diffusion rate by the surface modification, a drop of $\mathrm{MAPbI}_{3}$ precursor solution was placed at the end of two regular glass substrates (left in Fig. 2g) and two ITO substrates covered by PTAA (right in Fig. 2g), which had a same gap of $10 \mu \mathrm{m}$ filled with pure $\gamma$-butyrolactone (GBL) solvent, and they were hold at the temperature for crystal growth $\left(110^{\circ} \mathrm{C}\right)$ or room temperature. Driven by the concentration gradient, the precursor ions diffused in the GBL solution from the bottom side to the topside, and the diffusion of ions could be visualized by the color change of the solution with diffusion rate estimated by motion of front edge at the interface of pure GBL and perovskite precursor solution. As expected, the edge propagation was very slow $\left(0.01 \mathrm{~mm} \mathrm{~s}^{-1}\right)$ in the gap of two glass substrates, while was at least 100 -fold faster in the gap of two PTAA-covered ITO substrates (see Supplementary Video 1). We speculate that the much faster ion diffusion can be ascribed to the excellent lubrication effect of the nonwetting PTAA surface ${ }^{38}$. The nonwetting surface weakens the solvent-substrate interaction at the substrate surface, which will promote the diffusion of solvent. Therefore, the ions that form complexes with GBL molecules would not be dragged during the diffusion, as illustrated in Fig. 2f.

The fast ion transportation in the gap between two PTAAcovered substrates enables continuous growth of thin perovskite single crystals to a large area. The thin single crystals were grown on a PTAA-covered ITO substrate and the other PTAA-covered ITO substrate could be easily peeled off. The method is universally applicable to both iodide- and bromide-based perovskites. Figure $3 \mathrm{a}, \mathrm{b}$ shows the photographs of as-grown millimeter-size $\mathrm{MAPbI}_{3}$ and $\mathrm{MAPbBr}_{3}$ single crystals with thickness of $10-20 \mu \mathrm{m}$. As shown in Fig. 3c-e, thin $\mathrm{MAPbI}_{3}$ single crystals are obtained with tuned thickness from 10 to 40 $\mu \mathrm{m}$. For single crystals with thickness smaller than $10 \mu \mathrm{m}$, small- area multicrystals with lots of cracks were usually formed, which led to poor PCE for solar cells. The X-ray diffraction (XRD) pattern of a thin $\mathrm{MAPbI}_{3}$ single crystal in Fig. $3 \mathrm{f}$ shows only two sharp diffraction peaks which can be assigned to (200) and (400) planes, indicating that the crystal is (100) oriented. The corresponding rocking curve displays a small full-width at halfmaximum of $0.33^{\circ}$ for the (400) plane (Supplementary Fig. 1a), confirming the good quality of the thin single crystal. The $2 \mathrm{D}$ XRD shown in Supplementary Fig. 1b consists of scattered diffraction spots rather than a continuous arc, proving the singlecrystal character of the grown materials. XRD pattern of the powders scratched from the thin single crystals (inset of Fig. 3f) confirms the tetragonal structure of these crystals at room temperature. The top-view SEM image of the thin single crystal (Supplementary Fig. 2) shows that the surface of the thin single crystal is smooth. Figure $3 \mathrm{~g}$ shows the single-path absorption spectra of a single crystal with thickness of $10 \mu \mathrm{m}$ and a polycrystalline thin film with thickness of $500 \mathrm{~nm}$, which manifests an obvious redshift of the absorption spectrum edge from 790 to $810 \mathrm{~nm}$. The redshift of the absorption spectrum is general for other kinds of perovskites, such as $\mathrm{MAPbBr}_{3}$, $\mathrm{Cs}_{0.05}\left(\mathrm{FA}_{0.85} \mathrm{MA}_{0.15}\right)_{0.95} \mathrm{~Pb}\left(\mathrm{I}_{0.85} \mathrm{Br}_{0.15}\right)_{3} \quad$ (Supplementary Fig. 3) and $\mathrm{FAPbI}_{3}{ }^{24}$. The hole and electron mobility of the thin single crystals measured by the space charge limit current (SCLC) method (Supplementary Fig. 4) are $121 \pm 15 \mathrm{~cm}^{2} \mathrm{~V}^{-1} \mathrm{~s}^{-1}$ and $36.8 \pm 3.7 \mathrm{~cm}^{2} \mathrm{~V}^{-1} \mathrm{~s}^{-1}$, which are comparable to the values of bulk single crystals, indicating the good quality of the as-grown thin single crystals. The carrier recombination lifetime was measured by the transient photovoltaic (TPV) measurement under one-sun illumination (Fig. 4b). The obtained carrier lifetime is $2.2 \pm 0.5 \mu \mathrm{s}$, which is shorter than that of bulk single crystals measured by the same method, because the smaller volume of the thin single crystals increases the carrier density, thus increasing the bimolecular recombination. In addition, carriers in the thinner single crystals are more susceptible to surface recombination by a quicker transit to the crystal surface. Combining the measured mobility and lifetime, the hole diffusion 
a

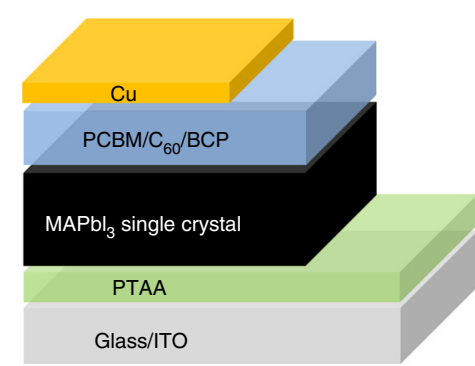

C

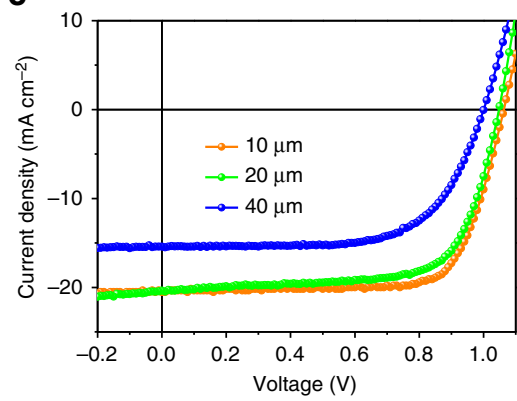

e

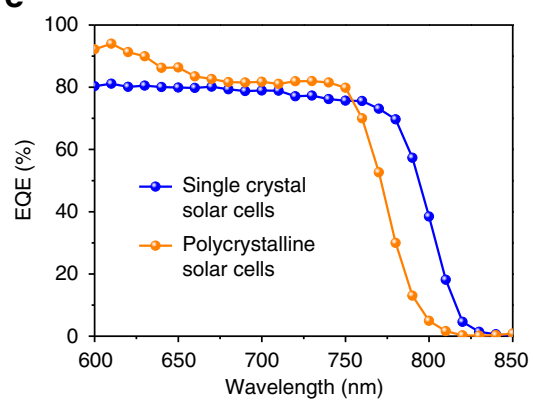

g

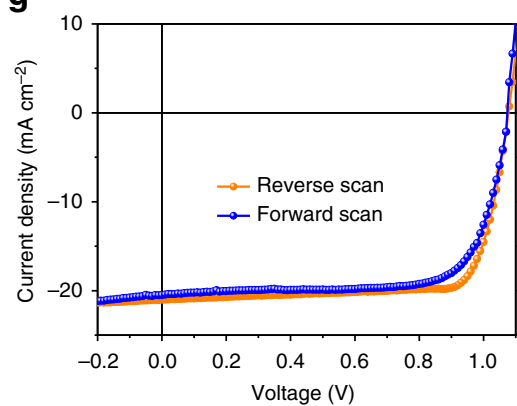

b

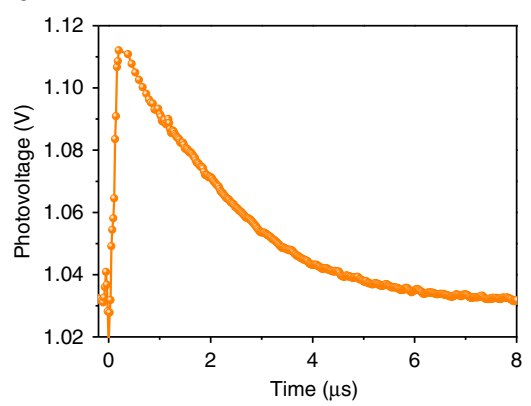

d

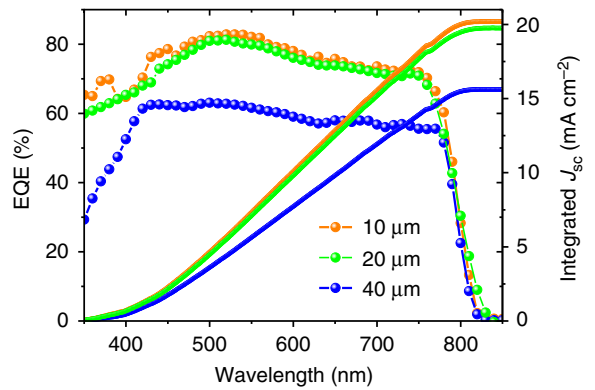

f

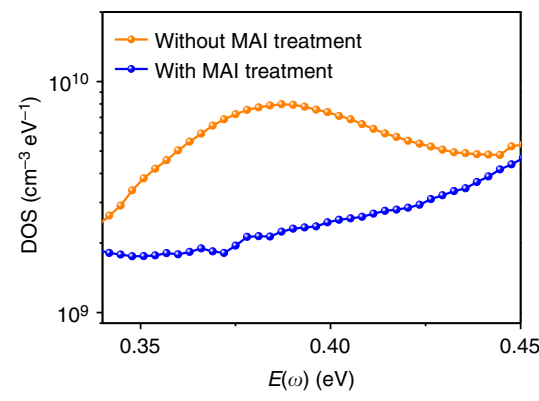

h

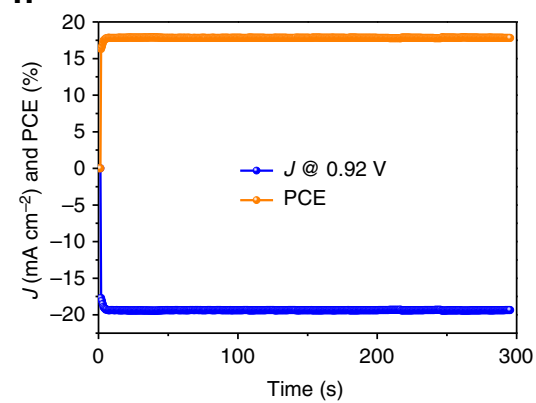

Fig. 4 Characterization of perovskite single-crystal solar cells. a Device structure of the single-crystal solar cells. b Transient photovoltaic curve of a singlecrystal solar cell under one-sun illumination. c Current density-voltage and $\mathbf{d}$ external quantum efficiency (EQE) curves and integrated current density of the optimal single-crystal solar cells using methylammonium lead triiodide thin single crystal with different thickness. e EQE of single-crystal solar cells and polycrystalline thin-film solar cells. $\mathbf{f}$ Trap density of states of the single-crystal solar cells before and after methylammonium iodide (MAI) treatment. $\mathbf{g}$ Current density-voltage curves of the single-crystal solar cells after MAI treatment measured under different scanning directions. $\mathbf{h}$ Stabilized $J_{\text {sc }}$ and PCE of single-crystal solar cells with MAI treatment. The thicknesses of the single crystals in Fig. e-h and polycrystalline thin film are 10 and $500 \mathrm{~nm}$, respectively

length and electron diffusion length under one-sun light are calculated to be 24.7 and $13.6 \mu \mathrm{m}$, respectively.

Perovskite single-crystal solar cells. Single-crystal solar cells with device structure of ITO/PTAA/MAPbI $3 /$ phenyl- $\mathrm{C}_{61}$-butyric acid methyl ester (PCBM)/ $\mathrm{C}_{60} /$ bathocuproine (BCP)/copper $(\mathrm{Cu})$
(Fig. 4a) were fabricated by depositing electron transport layers (ETLs) and metal electrodes on the top of the single crystals with the easy handling of ITO substrates. Figure $4 \mathrm{c}, \mathrm{d}$ shows the thickness-dependent J-V and EQE curves of the single-crystal solar cells. The highest device efficiency is obtained when using single crystals with thickness of $10 \mu \mathrm{m}$, which is consistent with the measured carrier diffusion length. The best device has a $J_{\mathrm{SC}}$ of 
$20.5 \mathrm{~mA} \mathrm{~cm}^{-2}$, a $V_{\text {OC }}$ of $1.06 \mathrm{~V}$, a FF of $74.1 \%$, and a PCE of 16.1\%. As shown in Fig. 4e, the EQE spectrum of this device shows an EQE cutoff at $820 \mathrm{~nm}$, corresponding to 20 -nm redshift compared to that of the polycrystalline thin-film devices. The larger redshift of EQE spectrum than the absorption spectrum should be attributed to the reflection of light by the electrode, which doubles the effective crystal thickness for light absorbing. The statistics of PCE, FF, $J_{\mathrm{SC}}$, and $V_{\mathrm{OC}}$ for devices using single crystals with thickness of $10 \mu \mathrm{m}$ are shown in Supplementary Fig. 6.

It is noted that thin single crystals may still have large surface charge traps ${ }^{40}$, though the bulk trap density should be very small. The surface charge traps are most likely generated from the loss of MAI when removing the single crystals out of the hot precursor solution ${ }^{41}$. Therefore, we treated the surface of the single crystals by spin coating MAI solution to reduce the surface charge trap density. This surface passivation effect was confirmed by the enhanced photoluminescence intensity (Supplementary Fig. 7). As shown in Fig. 4f, the surface trap density of the thin single crystals measured by thermal admittance spectroscopy is reduced by $2-5$-folds after MAI treatment. The PCE of the best device also increases to $17.8 \%$ with a $J_{\mathrm{SC}}$ of $21.0 \mathrm{~mA} \mathrm{~cm}^{-2}$, a $V_{\mathrm{OC}}$ of $1.08 \mathrm{~V}$, and a FF of $78.6 \%$ (Fig. $4 \mathrm{~g}$ ). The PCE of the device is confirmed by measuring the steady-state photocurrent output at the maximum power point (Fig. $4 \mathrm{~h}$ ). The increase of $J_{\mathrm{SC}}$ is consistent with the increase of the EQE values (Supplementary Fig. 8), and matches with the calculated $J_{\mathrm{SC}}$ from EQE. The corresponding statistics of PCE, FF, $J_{\mathrm{SC}}$, and $V_{\mathrm{OC}}$ are shown in Supplementary Fig. 9. By comparing with the calculated results in Fig. 1c, the $V_{\mathrm{OC}}$ is close to its predicted value of $1.14 \mathrm{~V}$, and the FF is close to what is observed in high-efficiency polycrystalline perovskite solar cells. The main limiting parameter of the present single-crystal solar cells is the smaller $J_{\mathrm{SC}}$ than the predicted value of $25.8 \mathrm{~mA} \mathrm{~cm}^{-2}$, which may be caused by the enhanced light reflection on the much flatter single-crystal surface (Supplementary Fig. 10a), in addition to charge collection loss caused by the incompletely passivated surface defects on both surfaces of the single crystals (Supplementary Fig. 10b). Addressing these issues will have potential to further increase the efficiency.

Finally, we evaluate the stability of the single-crystal solar cells. We have demonstrated that bulk single crystals showed better moisture stability than the polycrystalline thin films due to the absence of grain boundaries ${ }^{41}$. Therefore, the air stability of the single-crystal solar cells should be better than that of polycrystalline thin-film solar cells. To confirm this, the shell lifetime of the single-crystal solar cells in air is investigated. As shown in Supplementary Fig. 11, the PCE shows almost no change after the device was stored in air under dark for 30 days, which is better than the polycrystalline thin-film solar cells.

Conclusion. In conclusion, we demonstrated the use of a single crystal to broaden the photoresponse range of the perovskite solar cells without losing device photovoltage and fill factor. $\mathrm{MAPbI}_{3}$ thin single crystals were grown by a hydrophobic interfaceconfined lateral growth method, which yielded well control of crystal thickness at tens of micrometers. This work presents a new direction to further boost the efficiency and stability of perovskite solar cells. More efforts are needed to enhance the carrier diffusion length and passivate the surface nonradiative recombination center density in order to boost the device efficiency to SQ limit. The extended absorption can be attributed to the indirect bandgap absorption or Urbach band tail. Though there is some study that $\mathrm{MAPbI}_{3}$ has signature of indirect bandgap, there is no study whether the same conclusion can apply to $\mathrm{MAPbBr}_{3}$ or other perovskite materials yet. This study shows that these charges can actually be extracted out without being trapped.

\section{Methods}

Growth of perovskite thin single crystals. The perovskite thin single crystals were grown by a hydrophobic interface-confined lateral crystal growth method, as shown in Fig. 2. The PTAA film was prepared by spin coating $0.2 \mathrm{wt} \%$ PTAA solution in toluene at 4000 r.p.m. The as-prepared film was then thermally annealed at $100{ }^{\circ} \mathrm{C}$ for $10 \mathrm{~min}$. As illustrated in Fig. 2a, $1.5 \mathrm{M} \mathrm{MAPbI}_{3}$ solution in GBL was inserted into two ITO/PTAA substrates, which were then placed on a hot plate with temperature of $100^{\circ} \mathrm{C}$ for $1 \mathrm{~h}$. Subsequently, the temperature was elevated to 110 and $120^{\circ} \mathrm{C}$ to further promote the growth of the single crystals. Finally, the two substrates were separated to obtain the thin single crystals on ITO/ PTAA substrates. The temperature was slowly decreased to room temperature (about $2 \mathrm{~h}$ ) to ensure a good contact between PTAA and the single crystals.

Device fabrication. The device fabrication was completed by depositing the electron transport layer and electrode. PCBM solution of $1 \mathrm{wt} \%$ in chlorobenzene was spun on top of the thin single crystal and then annealed at $50^{\circ} \mathrm{C}$ for $10 \mathrm{~min}$. After that, C60 $(10 \mathrm{~nm}), \mathrm{BCP}(3 \mathrm{~nm})$, and $\mathrm{Cu}(80 \mathrm{~nm})$ were thermally evaporated in sequential order. The $\mathrm{C} 60$ and $\mathrm{BCP}$ were evaporated on the whole area of the surface of the single crystal. The $\mathrm{Cu}$ electrode was evaporated with a mask by covering the edge of the single crystal to avoid shorts. For MAI treatment, $0.5 \mathrm{mg}$ $\mathrm{ml}^{-1}$ MAI in IPA was spin coated on the single crystal before depositing PCBM and then annealed at $50^{\circ} \mathrm{C}$ for $10 \mathrm{~min}$.

Characterization. The absorption spectra were recorded by using an Evolution 201 UV-Visible Spectrophotometer. First, the transmission spectra of the thin single crystal and polycrystalline thin film were measured. Subsequently, the corresponding absorption spectra were calculated as Absorptance $=1$-Transmittance. The reflection spectrum was measured with the integrating sphere. The photocurrent density-voltage (J-V) curves of the devices were measured under AM 1.5 $\mathrm{G}$ irradiation $\left(100 \mathrm{~mW} \mathrm{~cm}^{-2}\right)$, which was produced by a xenon-lamp-based solar simulator (Oriel 67005, 150-W Solar Simulator). The light intensity was calibrated by a Schott visible-color glass-filtered (KG5 color-filtered) Si diode (Hamamatsu S1133) before photocurrent measurement. The J-V testing was performed with reverse scan direction at $0.1 \mathrm{~V} \mathrm{~s}^{-1}$ and sweep delay time was $10 \mathrm{~ms}$. The EQE was obtained using a Newport QE measurement kit by focusing a monochromatic beam of light onto the devices. The X-ray diffraction patterns were obtained by a Rigaku D/Max-B X-ray diffractometer in the Bragg-Brentano parafocusing geometry. A conventional cobalt target X-ray tube equipped in the diffracted-beam monochromator was set to $40 \mathrm{kV}$ and $30 \mathrm{~mA}$. The SEM images were taken from a Quanta 200 FEG environmental scanning electron microscope. In the TPV measurement, the single-crystal solar cells were illuminated by laser pulses $(337 \mathrm{~nm}, 4$ ns width from a SRS nitrogen laser) from the ITO side to generate a transient photovoltage signal which functions as a small perturbation to the background open-circuit voltage. The transient voltage signal was recorded by a 1-GHz Agilent digital oscilloscope.

Data availability. The data that support the findings of this study are available from the corresponding author on reasonable request.

Received: 9 June 2017 Accepted: 1 November 2017 Published online: 01 December 2017

\section{References}

1. Green, M. A., Ho-Baillie, A. \& Snaith, H. J. The emergence of perovskite solar cells. Nat. Photonics 8, 506-514 (2014).

2. Burschka, J. et al. Sequential deposition as a route to high-performance perovskite-sensitized solar cells. Nature 499, 316-319 (2013).

3. Snaith, H. J. Perovskites: the emergence of a new era for low-cost, highefficiency solar cells. J. Phys. Chem. Lett. 4, 3623-3630 (2013).

4. Liu, M., Johnston, M. B. \& Snaith, H. J. Efficient planar heterojunction perovskite solar cells by vapour deposition. Nature 501, 395-398 (2013).

5. Zhou, H. et al. Interface engineering of highly efficient perovskite solar cells. Science 345, 542-546 (2014).

6. Chen, W. et al. Efficient and stable large-area perovskite solar cells with inorganic charge extraction layers. Science 350, 944-948 (2015).

7. Yang, W. S. et al. High-performance photovoltaic perovskite layers fabricated through intramolecular exchange. Science 348, 1234-1237 (2015).

8. Li, X. et al. A vacuum flash-assisted solution process for high-efficiency largearea perovskite solar cells. Science 353, 58-62 (2016).

9. Yang, W. S. et al. Iodide management in formamidinium-lead-halide-based perovskite layers for efficient solar cells. Science 356, 1376-1379 (2017). 
10. Xiao, Z. et al. Thin-film semiconductor perspective of organometal trihalide perovskite materials for high-efficiency solar cells. Mater. Sci. Eng. R. Rep. 101, 1-38 (2016).

11. Stranks, S. D. et al. Electron-hole diffusion lengths exceeding 1 micrometer in an organometal trihalide perovskite absorber. Science 342, 341-344 (2013).

12. Wehrenfennig, C., Eperon, G. E., Johnston, M. B., Snaith, H. J. \& Herz, L. M. High Charge carrier mobilities and lifetimes in organolead trihalide perovskites. Adv. Mater. 26, 1584-1589 (2014).

13. Yin, W.-J., Shi, T. \& Yan, Y. Unusual defect physics in $\mathrm{CH}_{3} \mathrm{NH}_{3} \mathrm{PbI}_{3}$ perovskite solar cell absorber. Appl. Phys. Lett. 104, 063903 (2014).

14. Zdanowicz, T., Rodziewicz, T. \& Zabkowska-Waclawek, M. Theoretical analysis of the optimum energy band gap of semiconductors for fabrication of solar cells for applications in higher latitudes locations. Sol. Energy Mater. Sol. Cells 87, 757-769 (2005).

15. Jeon, N. J. et al. Compositional engineering of perovskite materials for highperformance solar cells. Nature 517, 476-480 (2015)

16. Eperon, G. E. et al. Formamidinium lead trihalide: a broadly tunable perovskite for efficient planar heterojunction solar cells. Energy Environ. Sci. 7, 982-988 (2014).

17. Noel, N. K. et al. Lead-free organic-inorganic tin halide perovskites for photovoltaic applications. Energy Environ. Sci. 7, 3061-3068 (2014).

18. Eperon, G. E. et al. Perovskite-perovskite tandem photovoltaics with optimized band gaps. Science 354, 861-865 (2016).

19. Motta, C. et al. Revealing the role of organic cations in hybrid halide perovskite $\mathrm{CH}_{3} \mathrm{NH}_{3} \mathrm{PbI}_{3}$. Nat. Commun. 6, 7026 (2015).

20. Brivio, F., Butler, K. T., Walsh, A. \& van Schilfgaarde, M. Relativistic quasiparticle self-consistent electronic structure of hybrid halide perovskite photovoltaic absorbers. Phys. Rev. B 89, 155204 (2014).

21. Hutter, E. M. et al. Direct-indirect character of the bandgap in methylammonium lead iodide perovskite. Nat. Mater. 16, 115-120 (2017).

22. Wang, T. et al. Indirect to direct bandgap transition in methylammonium lead halide perovskite. Energy Environ. Sci. 10, 509-515 (2017).

23. Saidaminov, M. I. et al. High-quality bulk hybrid perovskite single crystals within minutes by inverse temperature crystallization. Nat. Commun. 6, 7586 (2015).

24. Han, Q. et al. Single crystal formamidinium lead iodide $\left(\mathrm{FAPbI}_{3}\right)$ : insight into the structural, optical, and electrical properties. Adv. Mater. 28, 2253-2258 (2016).

25. Shi, D. et al. Low trap-state density and long carrier diffusion in organolead trihalide perovskite single crystals. Science 347, 519-522 (2015).

26. Dong, Q. et al. Lateral-structure single-crystal hybrid perovskite solar cells via piezoelectric poling. Adv. Mater. 28, 2816-2821 (2016).

27. Liu, Y. et al. Two-inch-sized perovskite $\mathrm{CH}_{3} \mathrm{NH}_{3} \mathrm{PbX}_{3}(\mathrm{X}=\mathrm{Cl}, \mathrm{Br}, \mathrm{I})$ crystals: growth and characterization. Adv. Mater. 27, 5176-5183 (2015)

28. Dang, Y. et al. Formation of hybrid perovskite tin iodide single crystals by topseeded solution growth. Angew. Chem. Int. Ed. 128, 3508-3511 (2016).

29. Peng, W. et al. Solution-grown monocrystalline hybrid perovskite films for hole-transporter-free solar cells. Adv. Mater. 28, 3383-3390 (2016)

30. Chen, Y.-X. et al. General space-confined on-substrate fabrication of thicknessadjustable hybrid perovskite single-crystalline thin films. J. Am. Chem. Soc. 138, 16196-16199 (2016)

31. Liu, Y. et al. Thinness- and shape-controlled growth for ultrathin singlecrystalline perovskite wafers for mass production of superior photoelectronic devices. Adv. Mater. 28, 9204-9209 (2016).

32. Liu, Y. et al. $120 \mathrm{~mm}$ single-crystalline perovskite and wafers: towards viable applications. Sci. China Chem. https://doi.org/10.1007/s11426-017-9081-3 (2017).

33. De Wolf, S. et al. Organometallic halide perovskites: sharp optical absorption edge and its relation to photovoltaic performance. J. Phys. Chem. Lett. 5, 1035-1039 (2014).

34. Jeon, N. J. et al. Solvent engineering for high-performance inorganic-organic hybrid perovskite solar cells. Nat. Mater. 13, 897-903 (2014).
35. Anderson, D. M., McFadden, G. B. \& Wheeler, A. A. Diffuse-interface methods in fluid mechanics. Annu. Rev. Fluid Mech. 30, 139-165 (1998).

36. Eperon, G. E., Burlakov, V. M., Docampo, P., Goriely, A. \& Snaith, H. J. Morphological control for high performance, solution-processed planar heterojunction perovskite solar cells. Adv. Func. Mater. 24, 151-157 (2014).

37. Zhumekenov, A. A. et al. The role of surface tension in the crystallization of metal halide perovskites. ACS Energy Lett. 2, 1782-1788 (2017).

38. Sendner, C., Horinek, D., Bocquet, L. \& Netz, R. R. Interfacial water at hydrophobic and hydrophilic surfaces: slip, viscosity, and diffusion. Langmuir 25, 10768-10781 (2009).

39. Bi, C. et al. Non-wetting surface-driven high-aspect-ratio crystalline grain growth for efficient hybrid perovskite solar cells. Nat. Commun. 6, 7747 (2015).

40. Yang, Y. et al. Top and bottom surfaces limit carrier lifetime in lead iodide perovskite films. Nat. Energy 2, 16207 (2017).

41. Dong, Q. et al. Electron-hole diffusion lengths $>175 \mu \mathrm{m}$ in solution-grown $\mathrm{CH}_{3} \mathrm{NH}_{3} \mathrm{PbI}_{3}$ single crystals. Science 347, 967-970 (2015).

\section{Acknowledgements}

The authors gratefully acknowledge financial support from Defense Threat Reduction Agency (HDTRA1-14-1-0030), Office of Naval Research (N00014-17-1-2163 and N00014-15-1-2713), and National Science Foundation (ECCS-1252623).

\section{Author contributions}

J.H. conceived the idea and supervised the project; Z.C., Q.D., and Y.L. grew the thin single crystal and fabricated the devices; C.B. carried out the tDOS measurement; Y.F. carried out the PL measurement; Y.L. fabricated the polycrystalline film solar cells; S.T. carried out the rocking curve measurement; Q.W. carried out the SEM measurement; X.X. carried out the TPV measurement; and Y.B. carried out the air stability test of the polycrystalline film solar cells. Y.D. carried out the XRD measurement. J.H., Z.C., and Q.D. wrote the manuscript, and all authors reviewed it.

\section{Additional information}

Supplementary Information accompanies this paper at https://doi.org/10.1038/s41467017-02039-5.

Competing interests: The authors declare no competing financial interests.

Reprints and permission information is available online at http://npg.nature.com/ reprintsandpermissions/

Publisher's note: Springer Nature remains neutral with regard to jurisdictional claims in published maps and institutional affiliations.

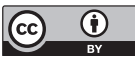

Open Access This article is licensed under a Creative Common Attribution 4.0 International License, which permits use, sharing, adaptation, distribution and reproduction in any medium or format, as long as you give appropriate credit to the original author(s) and the source, provide a link to the Creative Commons license, and indicate if changes were made. The images or other third party material in this article are included in the article's Creative Commons license, unless indicated otherwise in a credit line to the material. If material is not included in the article's Creative Commons license and your intended use is not permitted by statutory regulation or exceeds the permitted use, you will need to obtain permission directly from the copyright holder. To view a copy of this license, visit http://creativecommons.org/ licenses/by/4.0/.

(c) The Author(s) 2017 